Short communication

\title{
Anti-NMDA receptor encephalitis presenting with total insomnia - A case report
}

\author{
Inês Brás Marques *, Rute Teotónio, Catarina Cunha, Conceição Bento, Francisco Sales \\ Department of Neurology, Coimbra University Hospital, Praceta Prof. Mota Pinto, 3000-075 Coimbra, Portugal
}

\section{A R T I C L E I N F O}

\section{Article history:}

Received 9 September 2013

Received in revised form 7 October 2013

Accepted 21 October 2013

Available online 30 October 2013

\section{Keywords:}

Total insomnia

NMDAR encephalitis

Autoimmune encephalitis

Sporadic fatal insomnia

Video-EEG monitoring

Immunotherapy

\begin{abstract}
A B S T R A C T
Fatal insomnia (FI) is the first diagnosis to be considered by most neurologists when approaching a patient presenting with total insomnia followed by personality and cognitive changes, disturbance of alertness, autonomic hyperactivation and movement abnormalities.

We report the case of a 30 year-old male patient who presented with total insomnia followed by episodes of psychomotor restlessness resembling anxiety attacks. Twenty days later, he developed refractory convulsive status epilepticus with admission to Intensive Care Unit. He progressed to a state of reduced alertness and responsiveness, presenting periods of agitation with abnormal dyskinetic movements, periods of autonomic instability and central hypoventilation. Workup revealed antibodies against N-methyl-D-aspartate receptor (NMDAR). Immunotherapy treatment led to a very significant improvement with the patient presenting only slight frontal lobe dysfunction after one year of recovery.

To the best of our knowledge this is the first report of a patient with anti-NMDAR encephalitis first presenting with total insomnia. Our aim is to alert that anti-NMDAR encephalitis must be considered in the differential diagnosis of FI, especially in sporadic cases. Distinguishing the two conditions is very important as, contrarily to the fatal disclosure of FI, anti-NMDAR encephalitis is potentially reversible with adequate treatment even after severe and prolonged disease.
\end{abstract}

(c) 2013 Elsevier B.V. All rights reserved.

\section{Introduction}

When approaching a patient presenting with total insomnia followed by personality and cognitive changes, disturbance of alertness, autonomic hyperactivation and motor abnormalities, fatal insomnia (FI) is the first diagnosis to be considered. Although FI is more commonly a hereditary disease, this diagnosis may also be considered in patients without family history as sporadic cases have also been described [1].

In the present article we report the case of a young male patient who presented with total insomnia followed by behavioral changes, seizures and alertness decrease associated with abnormal movements, autonomic hyperactivation and central hypoventilation, which diagnostic workup revealed anti-N-methyl-D-aspartate receptor (NMDAR) encephalitis. A significant clinical improvement occurred after immunotherapy.

\subsection{Case report}

A 30 year-old male patient with unremarkable personal and familial past medical history presented with total insomnia of sudden onset. Four days later, he developed recurrent episodes of psychomotor restlessness, tachycardia, generalized tremors, diaphoreses and dyspnea with approximately 20 minute duration and was taken to the Emergency Room.

\footnotetext{
* Corresponding author. Tel.: + 351239400 448; fax: +351239822637.

E-mail address: inesmbmarques@gmail.com (I.B. Marques).
}

Alprazolam was prescribed for anxiety disorder, however total insomnia persisted, the episodes of restlessness increased in frequency, occurring 8 to 10 times per day, and the patient became irritable and aggressive. He was admitted to a Psychiatric Department two days later and medicated with olanzapine, diazepam, haloperidol and valproic acid with no clinical improvement. Twenty days later, he developed hyperthermia $\left(39.7{ }^{\circ} \mathrm{C}\right)$ and refractory generalized tonic-clonic seizures requiring anesthesia with propofol and midazolam. He was transferred to an Intensive Care Unit and empirical treatment with acyclovir, ceftriaxone, dantrolene and bromocriptine was started, considering the hypothesis of central nervous system infection or neuroleptic malignant syndrome. Blood workup including complete blood count, coagulation study, biochemistry and serological testing (Herpes simplex virus type 1 and type 2, Cytomegalovirus, Enterovirus, Echovirus, Adenovirus, Coxsackie virus, Coxiella burnetii, Toxoplasma gondii, Measles, Mumps, Treponema pallidum, Borrelia burgdorferi and Brucella) was unremarkable, as well as cerebrospinal fluid (CSF) cytochemical analysis, serological testing (Herpes simplex virus type 1 and type 2, Cytomegalovirus, Measles, Adenovirus, Coxsackie virus and Echovirus) and cultures. Brain computed tomography (CT) scan and magnetic resonance imaging (MRI) did not reveal any abnormal findings. Electroencephalogram (EEG) showed slow activity and sparse epileptic activity in the right frontal region. Several attempts to withdraw anesthetic treatment were performed during the following four weeks, but refractory convulsive status epilepticus always recurred. 
One month after status epilepticus onset, the patient was transferred to our hospital in order to perform continuous video-EEG monitoring. At admittance, EEG revealed diffuse slow activity and non-continuous focal seizures, so anesthetic discontinuation was tried once more. Fluctuation of alertness and diminished responsiveness were observed, with periods of total unresponsiveness alternating with periods of dissociative responses to stimuli, characterized by absent response to painful or visual stimuli but resistance to attempts of eyelid opening. Periods of total immobility and generalized hypertonia (Video 1) alternated with periods of agitation with abnormal movements, mainly involving the left upper limb and oromandibular muscles, with variable dyskinetic movements including tremor, choreoathetosis, dystonia and abnormal postures (Videos 2-4). Dyskinetic movements involving the trunk and the remaining limbs were also noticed although less frequently (Video 5). Periods of autonomic instability were identified consisting of tachycardia, arterial hypertension, diaphoresis and hyperthermia. Central hypoventilation was also present, with inability to suspend invasive ventilation after anesthetic withdrawal.

Video-EEG monitoring revealed diffuse asymmetric slowing with right preponderance and frequent rhythmic delta activity in the anterior regions of the right hemisphere (Fig. 1). Sleep patterns were absent. No correlation could be found between abnormal movements and EEG tracing, with most abnormal movements occurring without evidence of ictal patterns (Fig. 2, Video 4). Unequivocal ictal activity was infrequent and was either clinically silent or associated with movements similar to the movements observed in the absence of ictal patterns.

Diagnostic work-up was complemented with systemic autoimmune study (ANAs, anti-dsDNA, anti-SSA, anti-SSB, anti-Sm, anti-RNP, antiScl70, anti-Jo-1, p-ANCA, c-ANCA, anti-TPO and anti-tiroglobulin) and search for occult malignancy (tumor markers: CEA, CA19-9, CYFRA 21.1, NSE, $\beta$-HCG, PSA, alpha-fetoprotein; anti-neuronal antibodies: anti-Hu, Ri, Yo, amphiphysin, MA2, CV2; thorax X-ray; abdominal and testicular ultrasound), without relevant findings. CSF analysis was repeated revealing normal cytochemistry, negative 14.3 .3 protein and positive oligoclonal bands unmatched in serum. Autoimmune encephalitis antibody screening was requested, including antibodies against NMDAR, voltage-gated potassium channel (VGKC) complexes, gamma-aminobutyric acid type $\mathrm{B}\left(\mathrm{GABA}_{\mathrm{B}}\right)$ receptor and $\alpha$-amino-3hydroxy-5-methyl-4-isoxazolepropionic acid (AMPA) receptor. Considering the clinical suspicion of autoimmune encephalitis, high-dose intravenous (IV) methylprednisolone (MP) was administered for 5 days, followed by MP $1 \mathrm{mg} / \mathrm{kg} /$ day. No improvement occurred after 5 days of MP, so IV immunoglobulin (IVIG) was started ( $25 \mathrm{~g} /$ day, for 5 days). As the patient's clinical status continued progressively worsening after IVIG, plasma exchange (PE) on alternate days was tried. An improvement in alertness with short periods of interaction with the environment and brief periods of posterior rhythmic activity in EEG was observed after the third PE session. However, PE had to be discontinued after the fourth session due to hemoptysis secondary to hypofibrinogenemia, associated with severe anemia and hypotension. Nevertheless, 7 days later, after resolution of these medical complications, gradual improvement started with recovery of spontaneous ventilation and progressively longer periods of interaction with the environment.

Brain MRI was repeated and whole body positron emission tomography (PET) scan was performed, without significant findings. Brain ${ }^{18}$ F-FDG-PET scan revealed hypometabolism in the left hemisphere and relative hypermetabolism in the frontal and temporal regions of the right hemisphere (Fig. 3). Auto-antibody analysis identified antiNMDAR antibodies in serum and CSF.

Significant progressive clinical improvement occurred in the following months, with stabilization of the autonomic functions and normalization of the sleep-alertness cycle, including the presence of sleep patterns in the EEG, and subsequent recovery of motor skills and language. Behavioral changes, namely psychomotor restlessness and aggressiveness, were initially reported, but gradually resolved during the first four months of convalescence. Immunosuppressive therapy with azathioprine (50 mg/day) was started after immunotherapy withdrawal.

After one year, neuropsychological assessment reveals moderate frontal lobe dysfunction characterized by apathy, impulsivity and dysexecutive syndrome, and EEG shows persistence of slow activity in

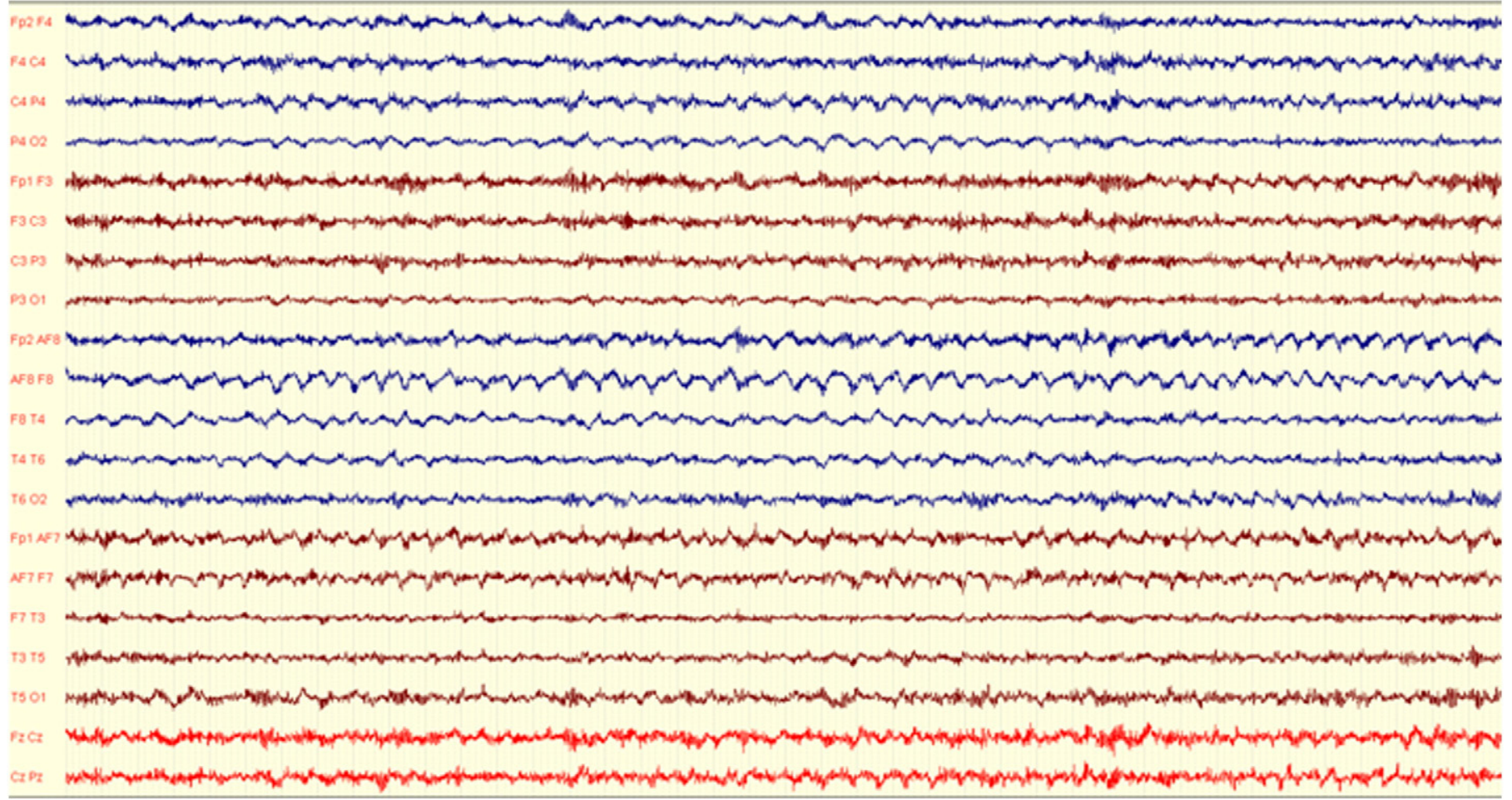

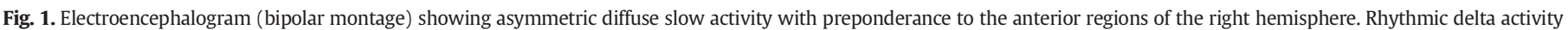
$(1.5 \mathrm{~Hz})$ is observed in the right frontal and temporal regions. 


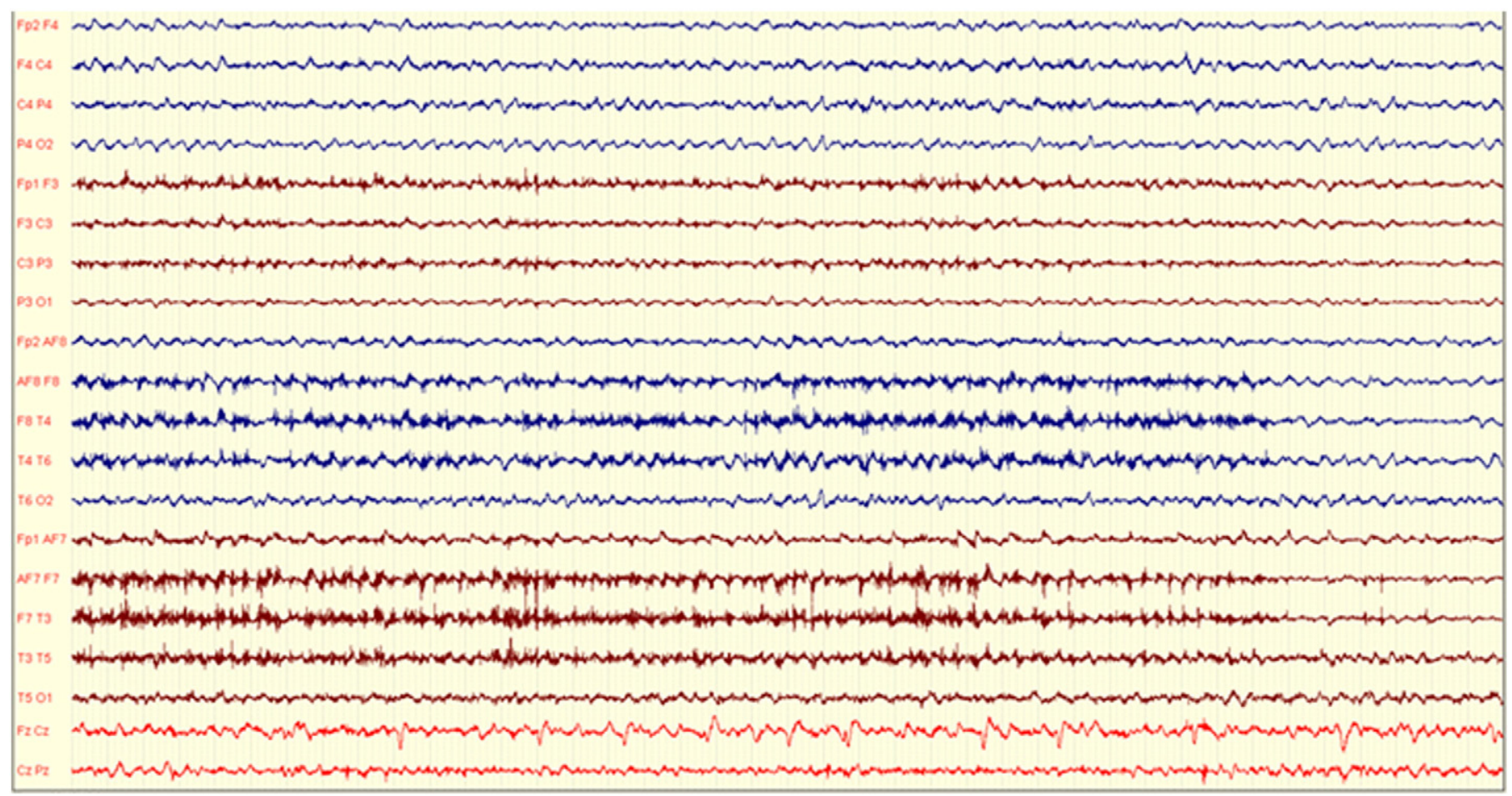

Fig. 2. Electroencephalogram (bipolar montage) during oromandibular and left upper limb dyskinetic movements (Video 4) revealing movement artifacts and no identifiable ictal pattern.

the anterior regions of the right hemisphere (Fig. 4). Whole Body PET scan was repeated without any relevant findings. Brain ${ }^{18} \mathrm{~F}$-FDG-PET scan shows significant improvement of brain metabolism (Fig. 5).

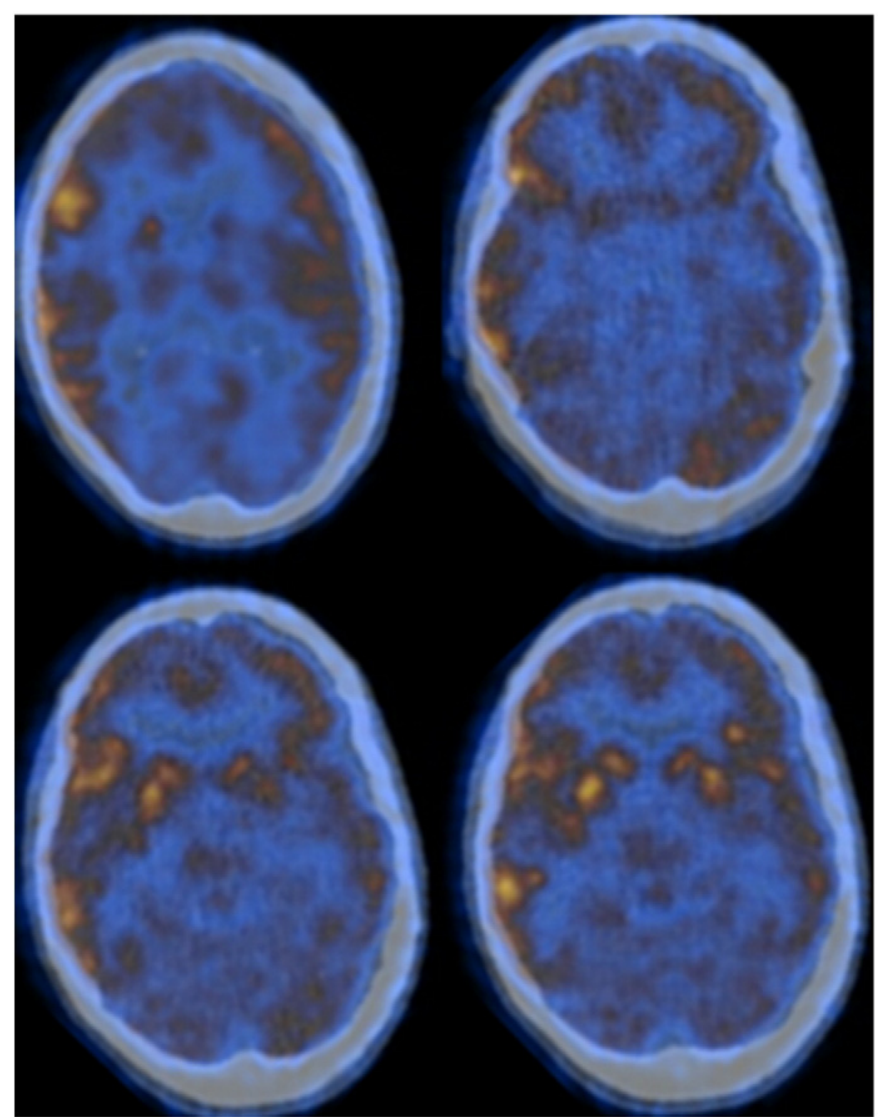

Fig. 3. Brain ${ }^{18}$ F-FDG PET showing diffuse hypometabolism and relative right frontotemporal hypermetabolism.

\section{Discussion}

Anti-NMDAR encephalitis has been described recently and its clinical features are still being defined. Several cases have been reported since the first description and it is now believed to be more common than initially thought and to be the most frequent type of antibodyassociated encephalitis $[2,3]$.

Our patient symptom progression, with psychiatric manifestations followed by seizures and diminished consciousness with abnormal movements, dysautonomia and central hypoventilation is in accordance with the described progression of anti-NMDAR encephalitis [4-6]. However, although insomnia is commonly described as an early presenting sign of the disease, the authors have not found another report of total insomnia as the first presentation of anti-NMDAR encephalitis. In the reported case, the presence of total insomnia resistant to benzodiazepines followed by behavioral symptoms, vigilance disturbance, autonomic hyperactivation and abnormal movements could resemble sporadic FI.

FI usually presents with a progressive decrease of sleep which culminates in total insomnia resistant to medication as benzodiazepines and barbiturates and accompanied by progressive disturbance of vigilance, attention and memory, personality changes, motor abnormalities consisting of peculiar oneiric behaviors (motor gestures which are believed to be related to a dream content) and, in some patients, autonomic hyperactivation, including arterial hypertension, slight evening pyrexia, diaphoresis, increased lacrimation and sialorrhea [7,8]. However, FI characteristically has a slower progression, with about five months of progressive sleep decrease and behavioral and psychiatric changes before the patient presents with total insomnia [1,9-12] and usually other motor abnormalities develop with disease progression including myoclonus, ataxia, pyramidal signs, dysarthria and dysphagia [7]. Seizures may occur in both conditions $[4,5,13]$.

In anti-NMDAR encephalitis, EEG usually reveals non-specific slow and disorganized activity and sporadic ictal patterns that evolve to a slow continuous rhythmic theta-delta activity [4-6,14]. Characteristically, no correlation is found between EEG activity and abnormal movements and these movements do not respond to antiepileptic treatment 


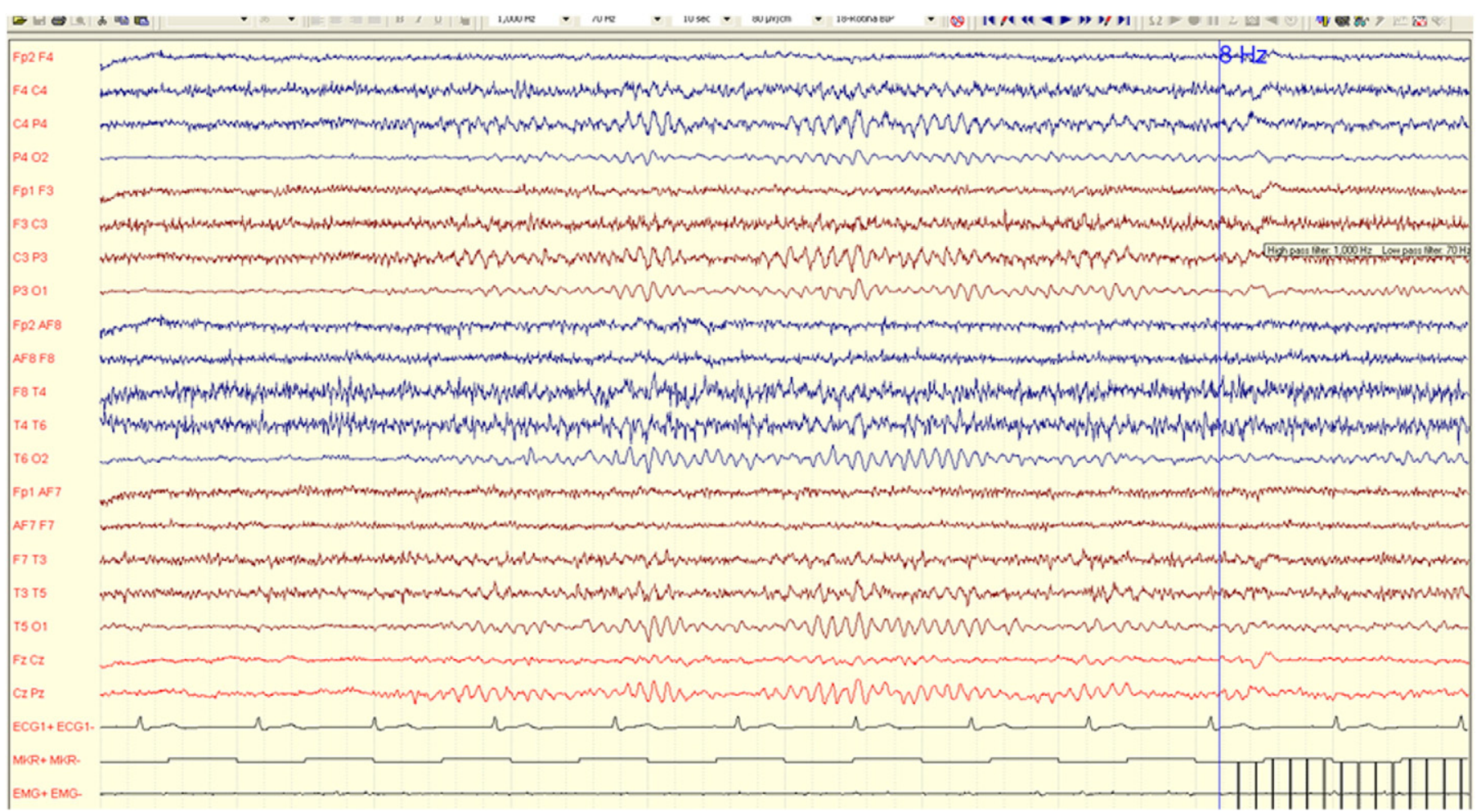

Fig. 4. EEG (bipolar montage) after one year, revealing persistence of slow activity in the right hemisphere anterior regions.

$[4,15,16]$. Therefore, continuous video-EEG monitoring may be important in differentiating epileptic seizures and nonepileptic abnormal movements, avoiding overtreatment with antiepileptics or anesthetics [16]. Brain MRI may be normal or present subtle and usually transient T2 or FLAIR signal hyperintensities in variable locations, usually without significant correlation with the clinical manifestations [4-6,17]. Brain PET scan abnormalities are non-specific and are usually variable, multifocal and involve cortical and subcortical regions [4,18-21]. Recently, a report analyzing brain PET scan of six patients with anti-NMDAR encephalitis detected a gradient of cerebral metabolism from frontotemporal to occipital areas [22]. The diagnosis is based on the identification of anti-NMDAR antibodies in serum or CSF [4,6,23].

In FI, EEG identifies progressive loss of sleep spindles and slow wave sleep, abnormal REM sleep stages and disruption of sleep cyclic organization $[8,24]$. The reported case alerts that similarly to FI, sleep patterns may be abnormal or even absent in anti-NMDAR encephalitis. Brain MRI

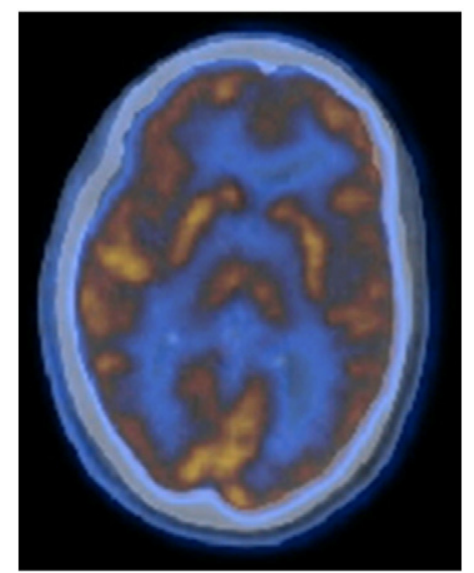

Fig. 5. Brain ${ }^{18} \mathrm{~F}$-FDG PET after one year showing significant improvement of brain metabolism. in patients with FI usually only presents non-specific cortical cerebral and cerebellar atrophy in late stages and brain PET scan typically shows thalamic and cingulated cortex hypometabolism [7]. In sporadic FI, 14.3.3 protein may be detected in CSF with an estimated positive predictive value of $95 \%$ [7].

Pathophysiologically, anti-NMDAR antibodies induce selective and reversible internalization of the post-synaptic NMDAR with decrease of glutamatergic function, essential to synaptic transmission and cerebral plasticity $[4,5,25]$. Current theories postulate that inactivation of GABAergic neurons leads to disinhibition of excitatory pathways resulting in frontostriatal syndrome and activation of brainstem central pattern generators inducing abnormal movements [4]. Hypoventilation is proposed to result from the direct effect of antibodies on the medullary-pontine respiratory network and dysautonomia from the effect of the antibodies on the dopaminergic, noradrenergic and cholinergic systems which have NMDAR [4].

FI is believed to result from a disturbance in the thalamolimbic system involved in the regulation of the wake-sleep cycle and other hormonal and circadian functions, resulting in a shift to persistent wake behavior and autonomic hyperactivation [7,8,24,26].

While FI currently has no treatment, presenting a fatal disclosure after 13 to 73 months [1,10-12], anti-NMDAR encephalitis is treatable, with about 75 to $81 \%$ of patients presenting significant improvement after treatment $[4,5,27]$. First-line treatment consists of immunotherapy (corticosteroids, IVIG and/or PE) and, when an associated neoplasm is found, tumor removal $[4,6,27]$.

NMDAR internalization by the antibodies is potentially reversible after antibody removal with immunotherapies [5,14], thus the neuronal dysfunction induced by the antibodies is potentially reversible even in cases of severe and prolonged disease. Therefore, treatment must always be performed irrespective of disease duration.

When no improvement occurs with first-line treatment and there is a confirmed diagnosis or a strong clinical suspicion of anti-NMDAR encephalitis, second-line treatment with rituximab and/or cyclophosphamide must be tried as it may improve patient outcome $[4,6]$. 
Clinical recovery is usually slow and occurs in the reverse order of symptom onset [4]. Social behavior and executive function recovery is usually prolonged and may be incomplete, with a significant proportion of patients with considerable recovery remaining with frontal dysfunction [4-6,28]. Relapses occur in 12 to $25 \%$ of patients [5,15,29,30], more commonly in patients without associated tumor, so immunosuppression with azathioprine or mycophenolate mofetil during at least 1 year after withdrawal of the initial immunotherapies and periodic tumor screening is advised in these patients [4-6].

In conclusion, this report alerts that anti-NMDAR encephalitis may first present with severe or even total insomnia, followed by behavioral and cognitive changes, alertness disturbance, abnormal movements and dysautonomia. As this presentation may resemble FI, anti-NMDAR encephalitis should be considered in the differential diagnosis of FI, especially in sporadic cases. The distinction between the two conditions is important, as, contrarily to the fatal disclosure of FI, anti-NMDAR encephalitis is potentially reversible with immunotherapies even in cases of severe and prolonged disease.

Supplementary data to this article can be found online at http://dx. doi.org/10.1016/j.jns.2013.10.034.

\section{Conflicts of interest}

All authors declare that they have no conflict of interest.

\section{References}

[1] Mastrianni JA, Nixon R, Layzer R, Telling GC, Han D, DeArmond SJ, et al. Prion protein conformation in a patient with sporadic fatal insomnia. $\mathrm{N}$ Engl J Med 1999;340:1630-8.

[2] Granerod J, Ambrose HE, Davies NW, Clewley JP, Walsh AL, Morgan D, et al. Causes of encephalitis and differences in their clinical presentations in England: a multicentre, population-based prospective study. Lancet Infect Dis 2010;10:835-44.

[3] Gable MS, Sheriff H, Dalmau J, Tilley DH, Glaser CA. The frequency of autoimmune Nmethyl-D-aspartate receptor encephalitis surpasses that of individual viral etiologies in young individuals enrolled in the California Encephalitis Project. Clin Infect Dis 2012;54:899-904.

[4] Dalmau J, Lancaster E, Martinez-Hernandez E, Rosenfeld MR, Balice-Gordon R. Clinical experience and laboratory investigations in patients with anti-NMDAR encephalitis. Lancet Neurol 2011;10:63-74.

[5] Dalmau J, Gleichman AJ, Hughes EG, Rossi JE, Peng X, Lai M, et al. Anti-NMDA-receptor encephalitis: case series and analysis of the effects of antibodies. Lancet Neurol 2008;7:1091-8.

[6] Peery HE, Day GS, Dunn S, Fritzler MJ, Prüss H, De Souza C, et al. Anti-NMDA receptor encephalitis. The disorder, the diagnosis and the immunobiology. Autoimmun Rev 2012;11:863-72.

[7] Montagna P, Gambetti P, Lugaresi E. Familial and sporadic fatal insomnia. Lancet Neurol 2003;2:167-76.

[8] Tinuper P, Montagna P, Medori R, Cortelli P, Zucconi M, Baruzzi A, et al. The thalamus participates in the regulation of the sleep-waking cycle: a clinico-pathological study in fatal familial thalamic degeneration. Electroencephalogr Clin Neurophysiol 1989;73:117-23.
[9] Kawasaki K, Wakabayashi K, Kawakami A, Higuchi M, Kitamoto T, Tsuji S, et al Thalamic form of Creutzfeldt-Jakob disease or fatal insomnia? Report of a sporadic case with normal prion protein genotype. Acta Neuropathol 1997;93:317-22.

[10] Yamashita M, Yamamoto T, Nishinaka K, Udaka F, Kameyama M, Kitamoto T. Severe brain atrophy in a case of thalamic variant of sporadic CJD with plaque-like PrP deposition. Neuropathology 2001;21:138-43.

[11] Piao YS, Kakita A, Watanabe H, Kitamoto T, Takahashi H. Sporadic fatal insomnia with spongiform degeneration in the thalamus and widespread PrPSc deposits in the brain. Neuropathology 2005;25:144-9.

[12] Capellari S, Parchi P, Cortelli P, Avoni P, Casadei GP, Bini C, et al. Sporadic fatal insomnia in a fatal familial insomnia pedigree. Neurology 2008;70:884-5.

[13] Montagna P, Cortelli P, Avoni P, Tinuper P, Plazzi G, Gallassi R, et al. Clinical features of fatal familial insomnia: phenotypic variability in relation to a polymorphism at codon 129 of the prion protein gene. Brain Pathol 1998;8:515-20.

[14] Jones KC, Benseler SM, Moharir M. Anti-NMDA receptor encephalitis. Neuroimaging Clin N Am 2013;23:309-20.

[15] Florance NR, Davis RL, Lam C, Szperka C, Zhou L, Ahmad S, et al. Anti-N-methyl-Daspartate receptor (NMDAR) encephalitis in children and adolescents. Ann Neurol 2009;66:11-8.

[16] Dericioglu N, Vural A, Acar P, Agayeva N, Ismailova V, Kurne A, et al. Antiepileptic treatment for anti-NMDA receptor encephalitis: the need for video-EEG monitoring. Epileptic Disord 2013;15:166-70.

[17] Dalmau J, Tüzün E, Wu HY, Masjuan J, Rossi JE, Voloschin A, et al. Paraneoplastic anti-N-methyl-D-aspartate receptor encephalitis associated with ovarian teratoma. Ann Neurol 2007;61:25-36.

[18] Maeder-Ingvar M, Prior JO, Irani SR, Rey V, Vincent A, Rosetti AO. FDG-PET hyperactivity in basal ganglia correlating with clinical course in anti-NDMA-R antibodies encephalitis. J Neurol Neurosurg Psychiatry 2011;82:235-6.

[19] Pillai SC, Gill D, Webster R, Howman-Giles R, Dale RC. Cortical hypometabolism demonstrated by PET in relapsing NMDA receptor encephalitis. Pediatr Neurol 2010;43:217-20.

[20] Maqbool M, Oleske DA, Huq AH, Salman BA, Khodabakhsh K, Chugani HT. Nove FDG-PET findings in anti-NMDA receptor encephalitis: a case based report. J Child Neurol 2011;26:1325-8.

[21] Chanson JB, Diaconu M, Honnorat J, Martin T, De Seze J, Namer IJ, et al. PET follow-up in a case of anti-NMDAR encephalitis: arguments for cingulate limbic encephalitis. Epileptic Disord 2012;14:90-3.

[22] Leypoldt F, Buchert R, Kleiter I, Marienhagen J, Gelderblom M, Magnus T, et al. Fluorodeoxyglucose positron emission tomography in anti-N-methyl-D-aspartate receptor encephalitis: distinct pattern of disease. J Neurol Neurosurg Psychiatry 2012;83:681-6.

[23] Vitaliani R, Mason W, Ances B, Zwerdling T, Jiang Z, Dalmau J. Paraneoplastic encephalitis, psychiatric symptoms, and hypoventilation in ovarian teratoma. Ann Neurol 2005;58:594-604.

[24] Sforza E, Montagna P, Tinuper P, Cortelli P, Avoni P, Ferrillo F, et al. Sleep-wake cycle abnormalities in fatal familial insomnia: evidence of the role of the thalamus in sleep regulation. Electroencephalogr Clin Neurophysiol 1995;94:398-405.

[25] Hughes EG, Peng X, Gleichman AJ, Lai M, Zhou L, Tsou R, et al. Cellular and synaptic mechanisms of anti-NMDA receptor encephalitis. J Neurosci 2010;30:5866-75.

[26] Lugaresi E, Tobler I, Gambetti P, Montagna P. The pathophysiology of fatal familial insomnia. Brain Pathol 1998;8:521-6.

[27] Titulaer MJ, McCracken L, Gabilondo I, Armangué T, Glaser C, Iizuka T, et al. Treatment and prognostic factors for long-term outcome in patients with anti-NMDA receptor encephalitis: an observational cohort study. Lancet Neurol 2013;12:157-65.

[28] Finke C, Kopp UA, Prüss H, Dalmau J, Wandinger KP, Ploner CJ. Cognitive deficits following anti-NMDA receptor encephalitis. J Neurol Neurosurg Psychiatry 2012;83:195-8.

[29] Irani SR, Bera K, Waters P, Zuliani L, Maxwell S, Zandi MS, et al. N-methyl-Daspartate antibody encephalitis: temporal progression of clinical and paraclinical observations in a predominantly non-paraneoplastic disorder of both sexes. Brain 2010;133:1655-67.

[30] Gabilondo I, Saiz A, Galán L, González V, Jadraque R, Sabater L, et al. Analysis of relapses in anti-NMDAR encephalitis. Neurology 2011;77:996-9. 